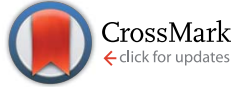

Cite this: Soft Matter, 2015, 11, 3125

\title{
Polymer fullerene solution phase behaviour and film formation pathways $\dagger$
}

\begin{abstract}
Rajeev Dattani and João T. Cabral*
We report the phase behaviour of polymer/fullerene/solvent ternary mixtures and its consequence for the morphology of the resulting composite thin films. We focus particularly on solutions of polystyrene (PS), $\mathrm{C}_{60}$ fullerene and toluene, which are examined by static and dynamic light scattering, and films obtained from various solution ages and thermal annealing conditions, using atomic force and light microscopy. Unexpectedly, the solution phase behaviour below the polymer overlap concentration, $c^{*}$, is found to be described by a simple excluded volume argument (occupied by the polymer chains) and the neat $\mathrm{C}_{60}$ /solvent miscibility. Scaling consistent with full exclusion is found when the miscibility of the fullerene in the solvent is much lower than that of the polymer, giving way to partial exclusion with more soluble fullerenes (phenyl-C61-butyric acid methyl ester, PCBM) and a less asymmetric solvent (chlorobenzene), employed in photovoltaic devices. Spun cast and drop cast films were prepared from $\mathrm{PS} / \mathrm{C}_{60} /$ toluene solutions across the phase diagram to yield an identical $\mathrm{PS} / \mathrm{C}_{60}$ composition and film thickness, resulting in qualitatively different morphologies in agreement with our measured solution phase boundaries. Our findings are relevant to the solution processing of polymer/fullerene composites (including organic photovoltaic devices), which generally require effective solubilisation of fullerene derivatives and polymer pairs in this concentration range, and the design of well-defined thin film morphologies.
\end{abstract}

Received 8th January 2015

Accepted 24th February 2015

DOI: $10.1039 / c 5 s m 00053 j$

www.rsc.org/softmatter
Careful studies of binary polymer/NP mixtures, typically with functionalised silica or gold NPs, ${ }^{6-10}$ have resulted in much controversy in terms of changes in polymer chain dimensions and (related) dispersion quality of the nanocomposites, which are generally cast from solution. Understanding solution miscibility, polymer conformation and NP association, and their evolution along film formation pathways is evidently required to fully exploit the potential of nanostructured composites in general.

Polymer and colloid solutions are often discussed ${ }^{\mathbf{1 1}}$ in terms of the relative dimensions of their constituents (viz. polymer radius of gyration $R_{\mathrm{g}}^{\text {poly }}$ and colloid $R_{\mathrm{C}}$ ), defining the so-called protein $\left(R_{\mathrm{C}} \ll R_{\mathrm{g}}^{\text {poly }}\right)$ and colloid $\left(R_{\mathrm{C}} \gg R_{\mathrm{g}}^{\text {poly }}\right)$ limits. Small NPs (or indeed fullerenes) may be expected to form solutions in the protein limit and cause $R_{\mathrm{g}}^{\text {poly }}$ to change, depending on the polymer-NP interactions in solution and their relative concentrations. Conversely, the addition of a polymer can cause crystallisation of the NP, as it is found in protein solutions and from which the term 'protein limit' is derived. ${ }^{12}$ Solutions in the colloid limit can be expected for large colloids and also for clusters, for instance of poorly dispersed NPs. Dispersed fullerenes have $R_{\mathrm{C}} \approx 0.5 \mathrm{~nm}$ which, for most polymer masses $M_{\mathrm{W}}$ (including those considered in this work), should result in solutions in the protein limit. Theoretically, multiple interactions between each polymer coil and colloidal particles must thus be considered, and polymers are generally treated as chains of connected spheres, each of the size of a colloidal
Department of Chemical Engineering, Centre for Plastic Electronics, Imperial College London, London, SW7 2AZ, UK. E-mail: j.cabral@imperial.ac.uk

$\dagger$ Electronic supplementary information (ESI) available. See DOI: $10.1039 / \mathrm{c} 5 \mathrm{sm} 00053 \mathrm{j}$ 
particle. Both homogeneous ${ }^{\mathbf{1 3 , 1 4}}$ and phase separated ${ }^{\mathbf{1 5 - 1 7}}$ solutions have been predicted theoretically. Experimentally, phase separation and crystallisation have been observed in a number of systems.,.$^{5,21}$ In all cases, the polymer was a linear polystyrene chain and the small colloid was either octa- $n$-propylsilsesquioxane $\left(R_{\mathrm{C}}=0.7 \mathrm{~nm}\right)$, crosslinked poly (ethyl methacrylate) $\left(R_{\mathrm{C}}=8-42 \mathrm{~nm}\right)$, hydroxyl functionalized silica nanoparticles $\left(R_{\mathrm{C}}=11 \mathrm{~nm}\right)$ or $\mathrm{C}_{60}$ fullerene $\left(R_{\mathrm{C}}=0.35 \mathrm{~nm}\right)$. For some systems, significant coil contraction $(\approx 50 \%)$ was found in the presence of high enough ( $\geq 8$ wt $\%)$ colloid concentration. ${ }^{18-21}$

In this work, we examine polymer/fullerene/solvent mixtures by comprehensively characterising the ternary solution phase behaviour and the resulting (binary) polymer/fullerene film morphologies. We select polystyrene (PS) as the polymer matrix, fullerene $\mathrm{C}_{60}$, and toluene as a model system; while PS is fully miscible in toluene across the entire composition range, the reported miscibility of $\mathrm{C}_{60}$ in toluene is only $0.32 \mathrm{wt} \%{ }^{22}$ The miscibility of $\mathrm{C}_{60}$ in PS is estimated to be $1 \mathrm{wt} \%$ or lower, ${ }^{23}$ although higher 'dispersibility' limits are found for rapidly precipitated $^{23,24}$ or spin cast composite films. ${ }^{25,26}$ This $\mathrm{PS} / \mathrm{C}_{60} /$ toluene system exhibits therefore highly asymmetric solvent interactions, in which the solubility of the PS is much greater than that of $\mathrm{C}_{60}$ in toluene. For comparison, in order to decrease asymmetry in solubility, we also evaluate solutions comprising a more miscible fullerene, phenyl-C61-butyric acid methyl ester (PCBM), in toluene, and a solvent exhibiting considerably higher PCBM solubility, chlorobenzene.

Previous studies on annealed binary $\mathrm{PS} / \mathrm{C}_{60}$ thin films have shown a rich morphological behaviour, ranging from (meta) stable to rapidly dewetting films, from homogeneous and uniform to phase separated (sparsely nucleated to spinodally correlated) morphologies, and various coupling effects between these. ${ }^{25-31}$ Under certain conditions, an enriched $\mathrm{C}_{60}$ layer was reported at the substrate interface, ${ }^{25,27,28}$ albeit not generally. ${ }^{30}$ Morphological control parameters were found to include the polymer mass $M_{\mathrm{W}}{ }^{26}$ fullerene content, ${ }^{29}$ substrate surface energy, ${ }^{25,30,31}$ film thickness, temperature, and even light exposure (due to fullerene photo-chemistry in both films ${ }^{30}$ and solutions ${ }^{32}$ ).

We now seek to measure the solution phase behaviour and quantify its impact on the film morphology, in both the as-cast and thermally annealed films. Our hypothesis is that films of identical composition and thickness can exhibit considerable morphological differences arising from both their initial solution state and film formation pathway. We therefore evaluate $R_{\mathrm{C}}$ and $R_{\mathrm{g}}^{\text {poly }}$ in solution by static (SLS) and dynamic light scattering (DLS) to gauge miscibility, and film morphology by optical (OM) and atomic force microscopy (AFM) to correlate, for the first time, the solution and film properties of these academically and technologically important polymer-fullerene mixtures.

\section{Experimental}

Fullerene $\mathrm{C}_{60}(99 \%+$ purity) was purchased from MER Corp, polystyrene of molecular mass $\left(M_{\mathrm{W}}\right) 65 \mathrm{~kg} \mathrm{~mol}^{-1}$ and a polydispersity index 1.1 from Polymer Laboratories Ltd (referred to as $65 \mathrm{k})$, and toluene (99.5\% purity) from VWR. The necessary masses of $\mathrm{C}_{60}$ and PS were weighed into the same vial and the required mass of toluene was added. The resulting $\mathrm{PS} / \mathrm{C}_{60} /$ toluene solutions were stored in the dark (as light exposure is known to cause fullerene aggregation ${ }^{32}$ ) and stirred for 48 hours. All solution concentrations can be found in Table S1. $\dagger$ Details of additional systems investigated, namely PS $M_{\mathrm{W}}$ from 18 to $775 \mathrm{~kg} \mathrm{~mol}^{-1}$, as well as PCBM fullerene and chlorobenzene solutions are provided in the ESI. $\dagger$

For static light scattering (SLS) a SOFICA instrument (Societé Francaise d'Instruments de Contröle et d'Analyses, Le Mesnil Saint-Denis, S. et 0., France), modified by SLS Systemtechnik (G. Baur, Denzlingen, Germany) was employed. The SLS is equipped with a $5 \mathrm{~mW}$ HeNe laser source $(\lambda=633 \mathrm{~nm})$ and a goniometer covering $27 \leq \theta \leq 105^{\circ}$, and yielding a wavenumber range from $7.1 \leq q \leq 23.7 \times 10^{-3} \mathrm{~nm}^{-1}$, calculated using $q=\frac{4 \pi n}{\lambda} \sin \frac{\theta}{2}$, where $n$ is the refractive index of the solvent. Experiments were carried out on $2 \mathrm{~mL}$ of solution in cylindrical quartz cells with $1 \mathrm{~cm}$ diameter (Hellma, Müllheim, Germany) and a stopper to prevent evaporation. The light exposure during the SLS measurement was kept below 3-5 minutes to minimise photo-oxidation of fullerenes. The photomultiplier was calibrated with a toluene (puriss. p.a. $\geq 99.7 \%$, Sigma Aldrich) standard. SLS data were treated with the standard Guinier approximation to obtain $R_{\mathrm{C}}$ :

$$
\ln R(q)=\ln R_{0}-\frac{q^{2} R_{\mathrm{C}}^{2}}{3}
$$

Dynamic light scattering (DLS) experiments were carried out with a Malvern Nano-S (Malvern Instruments Ltd, UK) with a fixed detector at $173^{\circ}$, corresponding to $q=2.96 \times 10^{-2} \mathrm{~nm}^{-1}$. All experiments were performed in sealed, rectangular quartz cuvettes (Hellma, Müllheim, Germany). To account for the multiple decays correlation functions were fit using a sum of exponential decays:

$$
g^{(1)}(q, t)=\sum_{i=1}^{N} a_{i}(q) \exp \left(-\Gamma_{i}(q) t\right)
$$

where $1 \leq i \leq 3$, and $\Gamma_{i}=D_{i} q^{2}$ from which the diffusion coefficient for the $i$ th mode, $D_{i}$, can be found. Using $R_{\mathrm{h}, i}=\frac{k_{\mathrm{B}} T}{6 \pi \eta D_{i}}$, where $k_{\mathrm{B}}, T$ and $\eta$ are Boltzmann's constant, temperature (K) and the viscosity of the solvent, respectively, the hydrodynamic radius of the $i$ th mode, $R_{\mathrm{h}, i}$, can be found.

Thin films were fabricated from the appropriate solution via spin coating onto $1 \mathrm{~cm}^{2}\langle 100\rangle$ silicon substrates (Compart Tech) which were surface treated with a Ultraviolet UV Ozone Cleaner (Novascan) for 15 minutes. The spin coating frequency (rpm) was varied as necessary in order to achieve the same film thickness from solutions of different PS concentrations (thickness calibration is shown in Fig. S2 $\dagger$ ). Prior to annealing, film thicknesses were measured with a UV-visible interferometer (Filmetrics, F20-UV) calibrated with a $\mathrm{SiO}_{2}$ wafer standard. All films obtained via spin casting reported here are $130 \pm 3 \mathrm{~nm}$ thick. Drop cast samples were prepared by casting $50-100 \mu \mathrm{L}$ of 
solution (the higher PS concentration solutions were cast with a lower volume to maintain an identical film thickness) onto $1 \mathrm{~cm}^{2} \mathrm{Si}$ substrates at $60{ }^{\circ} \mathrm{C}$ and dried for 30 minutes resulting in a thickness of $\approx 20 \mu \mathrm{m}$.

Polymer/fullerene composite films were first imaged with an Olympus BX41M-LED reflectance microscope equipped with an AVT GigE GX1050C camera. Image analysis was carried out using ImageJ (v1.46r). Brightness and condenser/focussing settings were varied for maximum contrast between polymerrich and fullerene-rich regions, intensity thresholded and a fast Fourier transform and/or particle analysis was carried out. Atomic force microscopy (AFM) (Innova, Bruker) is employed in tapping mode to quantify nanoscale structures and topography in thin polymer composite films. Image files were processed using Gwyddion (v2.31): the raw AFM data were imported, levelled by mean plane subtraction, a polynomial background was removed and lines were corrected by matching the height median; the minimum height for all images was set to zero.

\section{Results and discussion}

Previously, using DLS, SLS and small angle neutron scattering (SANS), we have demonstrated that PS chain dimensions remain unperturbed across the dilute to semi-dilute regimes $(0$ to $9.5 \mathrm{wt} \% \mathrm{PS}$ ) with $\mathrm{C}_{60}$ concentrations ranging from 0 to 0.96 $\mathrm{wt} \%$ and a molecular weight ranging from 20 to $1000 \mathrm{~kg} \mathrm{~mol}^{-1}$ (ref. 5). Specifically, we have quantified the hydrodynamic radius $R_{h}^{\text {poly }}$ obtained from DLS and the radius of gyration $R_{\mathrm{g}}^{\text {poly }}$ obtained by SLS and SANS in the dilute regime, as well as the correlation length $\xi$ obtained by SANS in semi-dilute solutions. The experimental results for this system are consistent with our previous findings. Fig. 1a shows DLS correlation functions obtained at a constant polymer concentration and increasing $\mathrm{C}_{60}$ loading. The first decay in $g^{(1)}(q, t)$ corresponds to the polymer coil whose dimensions are shown to remain unchanged across the composition range in Fig. 1b. However, an additional decay appears with increasing $\mathrm{C}_{60}$ loadings shown in Fig. 1a which we have found to correspond to fullerene clusters. ${ }^{5}$

SLS was used to probe $\mathrm{C}_{60}$ cluster formation by polymer addition in solution and thus gauge solution miscibility. We first measured the exact fullerene solubility limit for our $\mathrm{C}_{60}$ grade in toluene by stirring solutions of various concentrations for $48 \mathrm{~h}$, in the absence of light, at room temperature. We find a miscibility limit of $0.27 \mathrm{wt} \% \mathrm{C}_{60}$ in toluene, below the $0.32 \mathrm{wt} \%$ reported previously. ${ }^{22}$ This difference may be not only due to the purity of the fullerene of solvents employed, but also possibly by the measurement method and aggregation kinetics. We note that in ref. 22 the solubility was measured by dilution and filtration of a saturated $\mathrm{C}_{60}$ solution, while we have prepared fresh solutions at each composition which we have found to be more reproducible.

Typical SLS datasets are shown in Fig. 1d and e from which the fullerene cluster size dependence on the $\mathrm{C}_{60}$ (Fig. 1d) and PS (Fig. 1e) concentration is estimated. We find that increasing either eventually leads to $\mathrm{C}_{60}$ clustering beyond a compositionspecific threshold; for instance, increasing PS loading causes $\mathrm{C}_{60}$ cluster formation at lower $\mathrm{C}_{60}$ concentrations, as shown in Fig. 1f. At sufficiently long times and/or concentrations, macroscopic $\mathrm{C}_{60}$ become visible to the naked eye. A schematic illustrating these findings is shown in Fig. 1c.
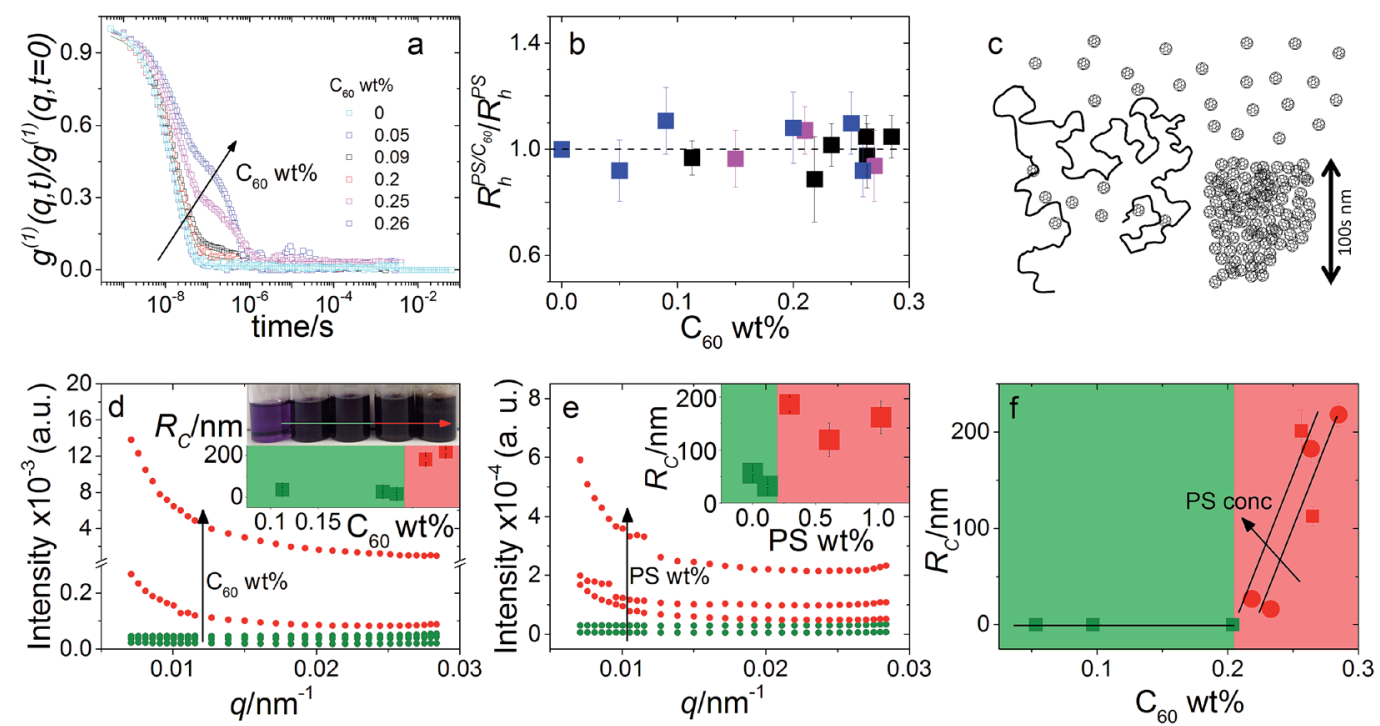

Fig. 1 (a) DLS correlation functions at $1.00 \pm 0.07$ wt\% PS with $\mathrm{C}_{60}$ from 0 to 0.26 wt\%. (b) Normalised $R_{\mathrm{h}}^{\mathrm{PS} / \mathrm{C}_{60}} / R_{\mathrm{h}}^{\mathrm{PS}}$ for $0.30 \pm 0.01$ ( $\square$ ), $0.6 \pm 0.1$ ( $\square$ ) and $1.00 \pm 0.07$ wt\% PS ( $\square$ ). We observe no change in $R_{\mathrm{h}}^{\mathrm{PS} / \mathrm{C}_{60}}$ with addition of $\mathrm{C}_{60}$, consistent with our previous findings. ${ }^{5}$ (c) Schematic depicting dispersed fullerenes and clusters on the order of 100's nm with unperturbed PS chains. (d and e) SLS of ternary solutions, (d) increasing the $\mathrm{C}_{60}$ concentration from 0.11 to $0.28 \mathrm{wt} \%$ and fixed $0.30 \pm 0.01 \mathrm{wt} \% \mathrm{PS}$ and (e) increasing the PS concentration from 0 to $1 \mathrm{wt} \%$ and fixed $0.25 \pm 0.01$ wt\% $C_{60}$. (f) Fullerene $R_{C}$ is dependent on the $C_{60}$ and PS concentration, shown with $0.30 \pm 0.01$ (•) and $1.00 \pm 0.07$ wt\% PS ( $\square$ and $\square$ ). Inset in (d) is an image of solution vials with increasing $\mathrm{C}_{60}$ loading and aggregates can be seen in the highlighted area. Inset in both (d) and (e) are estimates of $\mathrm{C}_{60}$ cluster sizes by a Guinier approximation. 
We next map the phase diagram by measuring a wide range of $\left(\mathrm{C}_{60}, \mathrm{PS}\right)$ concentrations in the dilute and semi-dilute regime of $65 \mathrm{k}$ PS, shown in Fig. 2. The green and red points and shaded areas define the miscible and two-phase regimes. We find that the addition of PS decreases $\mathrm{C}_{60}$ miscibility in a linear fashion, until a PS concentration $\left(<c^{*}\right)$ beyond which it becomes largely insensitive to further polymer addition. The initial linear decrease with polymer concentration suggests an expulsion of the fullerenes from the volume occupied by the PS chains, effectively increasing the fullerene concentration in a reduced available volume.

Given the (finite) miscibility of fullerenes in polystyrene, one could expect that the addition of a sufficiently small polymer fraction would not appreciably alter $\mathrm{C}_{60}$ miscibility in the ternary solution. This is not observed experimentally and, instead, the $\mathrm{C}_{60}$ miscibility drops from the outset of PS addition. In order to rationalise these findings we consider the simplest possible scenario: the $\mathrm{C}_{60}$ miscible fraction in solution is set by the volume remaining, $V_{\text {free}}$, after subtracting the volume occupied by the polymer chains, $V_{\text {occ}}$, from the total solution volume, $V_{\text {total }}$, yielding

$$
V_{\text {free }}=V_{\text {total }}-\alpha\left(N \frac{4 \pi R_{\mathrm{g}}{ }^{3}}{3}\right)
$$

where $V_{\text {occ }}$ is computed as $\left(N 4 \pi R_{\mathrm{g}}{ }^{3} / 3\right)$ where $N$ is the number density of chains (proportional to the concentration) and the dilute solution $R_{\mathrm{g}}^{\mathrm{PS}}$ is calculated by $R_{\mathrm{g}}(\mathrm{nm})=1.25 \times 10^{-5}$ $M_{\mathrm{W}}{ }^{0.595}$ in toluene. ${ }^{33}$ Effectively, the fullerene concentration is assumed to increase in the remaining $V_{\text {free }}$ and we consider the miscibility of $\mathrm{C}_{60}$ in toluene to remain unchanged. The scaling pre-factor $\alpha$ sets the gradient of the line shown in grey in Fig. 2 . We obtain a best fit for $\alpha=0.74$. Incidentally, we note that this value corresponds to the close packing of equal (impenetrable) spheres. Broadly speaking, the various estimates for $c^{*}$ rest on

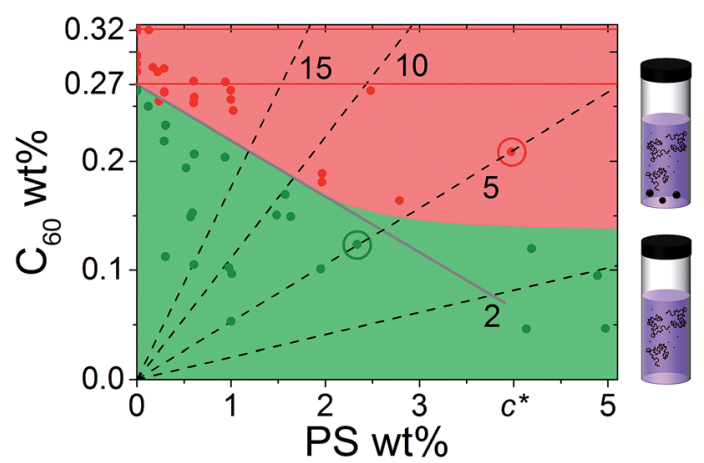

Fig. 2 Phase diagram of $P S / C_{60} /$ toluene solutions showing $C_{60}$ aggregation at 0-5 wt\% PS. Homogeneous (๑) and precipitated (๑) solutions are shown. The calculated solubility limit $(-)$ (described in the text) agrees well with the observed homogeneous and precipitated phases, shown in green and red, respectively. Red lines indicate neat $\mathrm{C}_{60} /$ toluene miscibility, previously reported $(0.32 \mathrm{wt} \%)^{22}$ and found in this study (0.27 wt\%). Dashed lines indicate the constant $\mathrm{PS} / \mathrm{C}_{60}$ ratio in the film, with the $\mathrm{C}_{60}$ mass\% noted. The circled data points, along the $\mathrm{PS}-5 \mathrm{wt} \% \mathrm{C}_{60}$ line, indicate solutions employed in the thin and bulk films investigated below. similar volume estimates ${ }^{\mathbf{3 4 , 3 5}}$ of single chain overlap and agreement with experimental data is often not quantitative, ${ }^{36}$ as the semi-dilute crossover is broad. Beyond $\sim \frac{2}{3} c^{*}$ the concentration of molecularly dispersed $\mathrm{C}_{60}$ appears to level off to the value between 0.12 and $0.15 \mathrm{wt} \%$. This result agrees with previous estimates of the finite miscibility of $\mathrm{C}_{60}$ in polystyrene $^{23,24}$ which we have estimated to be $<1 \mathrm{wt} \% \mathrm{C}_{60} \cdot{ }^{23}$ As expected, at sufficiently high polymer concentration, when chain overlap begins, fullerene miscibility no longer correlates with the simple volume estimate extrapolated from dilute chain dimensions. The asymptotic fullerene miscibility value should now be governed by the binary thermodynamic interaction with the host polymer matrix.

Given the strong power-law dependence of the volume occupied by single chains (and $c^{*}$ ) on polymer mass $M_{\mathrm{W}}$, we have carried out selected experiments by varying PS $M_{\mathrm{W}}$ (presented in Fig. $\mathrm{S} 1 \dagger)$. As expected from eqn (3), increasing $M_{\mathrm{W}}$ decreases $c^{*}$ and the miscibility of $\mathrm{C}_{60}$ decreases at a faster rate by addition of PS, as chains overlap 'earlier' (i.e. at a lower concentration). The slope $\alpha$ remained consistent with the value found above. We are thus surprised that this simple scaling model yields remarkable agreement with our data.

We can thus summarise our findings for $\mathrm{PS} / \mathrm{C}_{60} /$ toluene ternary solutions as follows: (i) increasing PS wt\% increases the $\mathrm{C}_{60}$ aggregate size (at constant solution age); (ii) the onset of $\mathrm{C}_{60}$ aggregation depends on the PS concentration with higher PS loadings inducing $\mathrm{C}_{60}$ aggregation at lower $\mathrm{C}_{60}$ concentrations; (iii) at a fixed mass, increasing the $M_{\mathrm{W}}$ of PS chains decreases the miscibility limit of $\mathrm{C}_{60}$; (iv) a simple volume argument below $c^{*}$ describes the trend observed for the miscibility of $\mathrm{C}_{60}$ by polymer addition of various $M_{\mathrm{W}}$.

We next consider the effect of ternary solution behaviour on the resulting $\mathrm{PS} / \mathrm{C}_{60}$ film morphology, both for thin and bulk films, both as-cast and thermally annealed. Increasing the polymer content generally increases the film thickness obtained by spin coating, carefully coupling the rpm and PS concentration allows films of identical thickness to be prepared from solutions with a range of solid contents. Our calibration curve for $\mathrm{PS} / \mathrm{C}_{60} /$ toluene is shown in Fig. S2. $\dagger$ Maintaining the PS to $\mathrm{C}_{60}$ ratio constant, indicated by the dashed lines in Fig. 2, nominally identical films can be prepared. Illustrative $5 \% \mathrm{C}_{60} / \mathrm{PS}$ films, on both sides of the miscibility boundary, are indicated by red and green circles in Fig. 2. We investigate the effects of solution age, thermal annealing and drying time.

\subsection{Solution age}

Over time, $\mathrm{C}_{60}$ within solutions in the precipitated regime (red) is found to aggregate. Fig. 3 compares the morphology of films which are nominally identical: $5 \% \mathrm{C}_{60} / \mathrm{PS}$ and $130 \mathrm{~nm}$ thick. Films cast from the immiscible (red) region yield $\mathrm{C}_{60}$ clusters whose number (density) increases with solution age (up to 5 days shown here). By contrast, films cast from the miscible (green) region yield uniform films regardless of solution age. This behaviour was confirmed along other fixed relative $\mathrm{C}_{60} / \mathrm{PS}$ composition lines (e.g. Fig. S3†). 


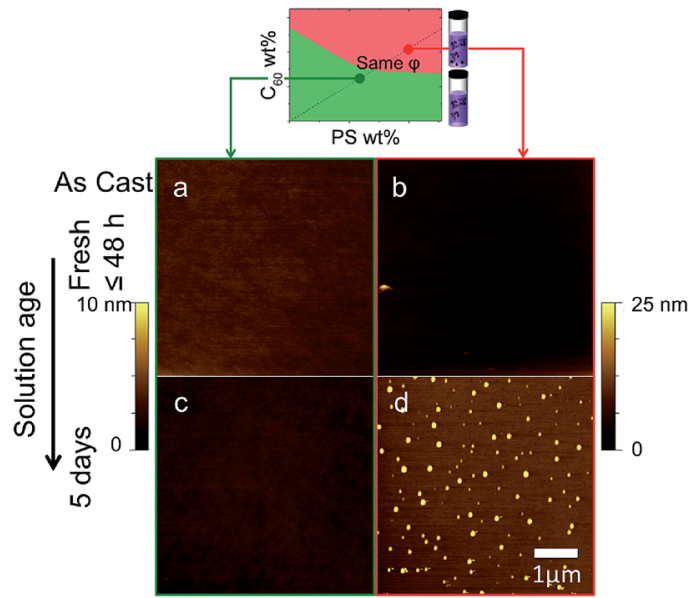

Fig. 3 Effect of solution age on spun cast thin films, across the miscibility boundary. AFM topography images of $5 \% \mathrm{C}_{60} / \mathrm{PS} 130 \mathrm{~nm}$ ascast thin films prepared from ( $a$ and $b$ ) a freshly made solution and (c and d) a solution aged for 5 days.

\subsection{Thermal annealing}

Annealing of thin films prepared from the two solution states, across the phase boundaries, yielded strikingly different morphologies shown in Fig. 4. Since the miscibility of $\mathrm{C}_{60}$ in PS is relatively low $\left(<1 \% \operatorname{mass}^{23}\right)$, one expects films cast for

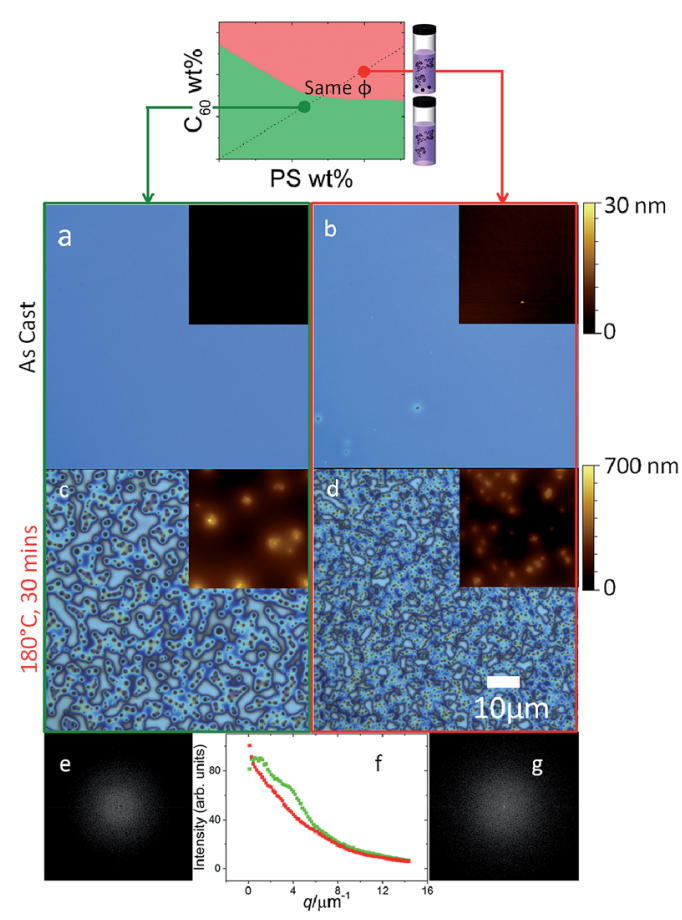

Fig. 4 Effect of thermal annealing $\left(180^{\circ} \mathrm{C}, 30 \mathrm{~min}\right)$ on the morphology of spun cast $5 \% \mathrm{C}_{60} / \mathrm{PS} 130 \mathrm{~nm}$ thin films prepared from solutions in the ( $a$ and $c$ ) homogeneous and ( $b$ and d) precipitated regimes. FFTs of (c) and (d) are shown in (e) and (g), respectively, whose radial profiles are compared in ( $f)$. Insets in $(a-d)$ show AFM scans of the corresponding films, with an image width of $10 \mu \mathrm{m}$; the height scale bars are $30 \mathrm{~nm}$ in ( $a$ and b) and $700 \mathrm{~nm}$ in (c and d). solutions with a higher $\mathrm{C}_{60}$ content to yield phase segregated morphologies ${ }^{29}$ upon thermal annealing above the glass transition temperature $T_{\mathrm{g}}$ of the mixtures (between 104 and $108{ }^{\circ} \mathrm{C}^{23}$ ). As seen previously (Fig $3 \mathrm{a}$ and b), as-cast films prepared from homogenous solutions (green) are uniform, whereas those from precipitated solutions (red) exhibit micronsized aggregates. Upon annealing, a characteristic 'spinodal clustering ${ }^{26,29}$ morphology can be observed in the film prepared from the homogeneous solution (Fig. 4c), whereas the annealed morphology of films prepared from the precipitated solution is markedly different, exhibiting more and, on average, smaller $\mathrm{C}_{60}$ clusters. The seed clusters from solution appear thus to alter the fullerene cluster formation in films, which seems dominated by fast nucleation (in addition to growth of seed clusters). Representative Fast Fourier Transforms (FFTs) show that the dominant length scale of films cast from homogeneous solutions (seen by a shoulder in the structure factor, green) is lost due to the polydispersed morphology of films cast from metastable solutions (in red).

\subsection{Drying timescales and film thickness}

We next consider the effect of the film casting method on the resulting film morphology. Given that the drying timescale of spin coating is relatively short (a few seconds), the 'as-cast' films obtained are found to quench morphologies closely matching the expectations from the solution state. As such, films spun cast from the miscible region of the solution phase diagram appear homogenous, even though their composition exceeds the miscibility limit of $\mathrm{C}_{60}$ in PS melts, since below $T_{\mathrm{g}}$. By contrast, films drop cast from solution may take up to $30 \mathrm{~min}$ to dry (above room temperature) and will thus explore the same drying pathway, across the miscibility limit, at a much slower rate. Drop casting from heterogeneous (red) solutions, should thus differ from spin casting, by allowing a slow crossing of the phase boundaries in addition to the growth of seed $\mathrm{C}_{60}$ clusters. The common drying pathway is shown in Fig. 5 and maintains a constant PS to $\mathrm{C}_{60}$ ratio throughout, and thus yields identical film compositions (and thickness through judicious choice of casting parameters).

The slower drop casting yields dramatically different composite morphologies, shown in Fig. 5e-h. Films cast from the homogeneous (green) solutions yield few $\sim 1 \mu \mathrm{m}$ clusters, as seen by optical microscopy in Fig. 5e, that nucleated during drying through the heterogeneous pathway. Upon thermal annealing above $T_{\mathrm{g}}$, diffuse $\mathrm{C}_{60}$ clusters of several $\mu \mathrm{m}$ forms (Fig. 5g), with a structure reminiscent of bundles with a ramified internal structure. Large $(\sim 100 \mu \mathrm{m})$ fractal fullerite $\mathrm{C}_{60}$ structures from toluene can be obtained under specific slow evaporation conditions ${ }^{37}$ suggesting that the slow evaporation and a higher viscosity of PS/toluene drop cast solutions yield these 'tumbleweed' structures. Directional assembly of $\mathrm{C}_{60}$ in solution with rod-like (and other) features, ${ }^{38,39}$ and for polymer/ colloid mixtures ${ }^{40}$ have been observed previously. By contrast, films drop cast from aggregated solutions (red) yield large (10's $\mu \mathrm{m}) \mathrm{C}_{60}$ compact crystalline (and birefringent) clusters regardless of annealing, as shown in Fig. $5 \mathrm{f}$ and h (and S4†), 


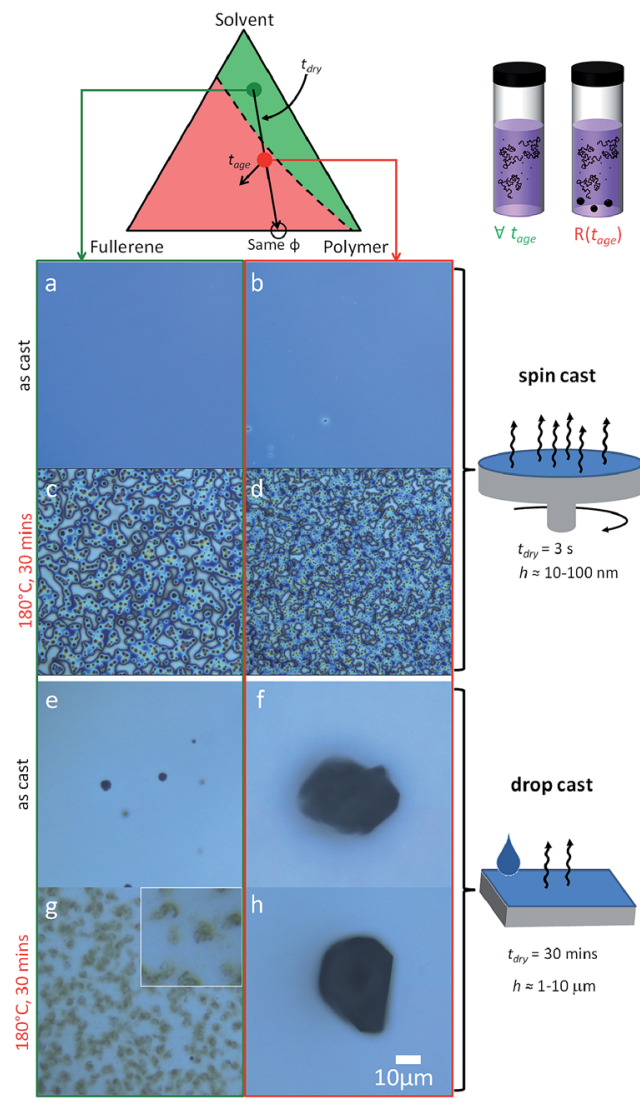

Fig. 5 Effect of drying time along the same film formation pathway. (a-d) $5 \mathrm{wt} \% \mathrm{C}_{60} / \mathrm{PS} 130 \mathrm{~nm}$ thin films fabricated via spin casting yield either an ordered (c) or disordered (d) morphology upon annealing. (e-h) $5 w t \% \mathrm{C}_{60} / \mathrm{PS} \approx 20 \mu \mathrm{m}$ thick drop cast films result in (e) sparse fullerene clusters nucleated during drying from homogeneous solutions (green), or (f) large precipitates seeded within the precipitated solution (red) that grow during slow drying. Upon thermal annealing, (g) clusters with a ramified internal structure form from homogeneous solutions and (h) large clusters, similar to those of as-cast films from the precipitated solution. Image width of inset in (g) is $10 \mu \mathrm{m}$. Image analysis is shown in Fig. $\mathbf{S 4} . \dagger$

suggesting that solution seed clusters grow extensively during the slow drying period and thus thermal annealing has no incremental (nucleation and growth) effect. The controlled growth of $\mathrm{C}_{60}$ micron-sized crystals with various shapes and crystal structures using solvent mixtures has been demonstrated (e.g. ref. 41). The drying pathway, across both sides of the phase boundary, has evidently a significant impact and potential on tuning the morphology of these polymer/fullerene composites.

\section{Conclusions}

We examined the ternary solution phase behaviour of a model $\mathrm{PS} / \mathrm{C}_{60} /$ toluene mixture to elucidate the effect of addition of a polymer to the miscibility of $\mathrm{C}_{60}$, as well as the effect of $\mathrm{C}_{60}$ on the polymer conformation. As we have shown previously ${ }^{5}$ and corroborated with this work, the conformation of PS remains unperturbed across the dilute and semi-dilute regimes. By contrast, the addition of PS to $\mathrm{C}_{60} /$ toluene solutions linearly decreases the miscibility limit of $\mathrm{C}_{60}$ in the mixture compared to that in neat toluene. We find that a simple volume argument assuming that the $\mathrm{C}_{60}$ concentration effectively increases by subtraction of the volume occupied by single PS chains describes remarkably well the experimental observations below $c^{*}$ for several $M_{\mathrm{W}}$. We find that the $\mathrm{C}_{60}$ miscibility eventually becomes largely insensitive to further PS addition between 0.12 and $0.15 \mathrm{wt} \% \mathrm{C}_{60}$, which is compatible with the estimate of $<1 \%$ wt for $\mathrm{PS} / \mathrm{C}_{60}$ (binary) mixtures. ${ }^{23}$

To assess the generality of our findings, we examined PS/ toluene solutions with a more soluble fullerene (PCBM), and PS/ PCBM solutions with a better solvent for the fullerene: chlorobenzene. These results are summarised in Fig. S5 and S6. $\dagger$ In short, we find that the phase boundaries vary qualitatively in a similar manner, i.e. a linear decrease in fullerene miscibility upon polymer addition. However, the volume estimate with $\alpha \simeq 0.74$ (reminiscent of hard sphere packing) was only applied to $\mathrm{PS} / \mathrm{C}_{60} /$ toluene which we attribute to the large asymmetry in the solubility of PS and $\mathrm{C}_{60}$ in toluene. As this asymmetry decreases (e.g. with PCBM and further with chlorobenzene), the miscibility reduction becomes less sensitive to polymer addition, effectively reducing $\alpha$ (all the way to $\simeq 0$ ). Fullerene miscibility and aggregation appears thus highly dependent on the relative magnitude of the interactions between the solution components.

We have considered $\mathrm{PS} / \mathrm{C}_{60}$ film morphologies obtained for identical compositions and thicknesses but formed through different solution pathways. In agreement with the measured phase diagram, we observe films that are rapidly formed by spin casting from the homogeneous region to be uniform, while those cast from above the miscibility line to contain clusters, that depend on solution age. Upon thermal annealing, these also yield different morphologies indicating opportunities for tuning the film morphology by controlling the solution properties, which have not been previously recognised. Slow drying of thick $(>\mu \mathrm{m})$ films by drop casting has a proportionally larger effect on the resulting morphology (of nominally identical films) by increasing the timescale for fullerene aggregation across the phase boundaries.

Our findings highlight the importance of understanding solution properties of polymer/fullerene (and polymer/nanoparticle) composites in a range of practical applications, including fullerene-based solar cells and advanced coatings that are solution processed.

\section{Acknowledgements}

The authors thank the Engineering and Physical Sciences Research Council (EPSRC) for funding (EP/G037515/1), the Royal Society (UK) for an International Exchange award, and the Institute for Molecular Engineering (IME) at the University of Chicago for generous hospitality of JTC. We thank Klaus Huber (Universität Paderborn, Germany) and Jack Douglas (NIST, Maryland) for many thought provoking and useful discussions. 


\section{References}

$1 \mathrm{~J}$. Keddie and A. F. Routh, Fundamentals of Latex Film Formation - Processes and Properties, Springer Laboratory, 2010.

2 J. Rivney, S. C. B. Mannsfeld, C. E. Miller, A. Salleo and M. F. Toney, Chem. Rev., 2012, 5488-5519.

3 B. C. Thompson and J. M. J. Fréchet, Angew. Chem., Int. Ed., 2008, 47, 58-77.

4 M. T. Dang, L. Hirsch and G. Wantz, Adv. Mater., 2011, 23, 3597-3602.

5 R. Dattani, R. Michels, A. J. Nedoma, R. Schweins, P. Westacott, K. Huber and J. T. Cabral, Macromolecules, 2014, 47, 6113-6120.

6 A. I. Nakatani, W. Chen, R. G. Schmidt, G. V. Gordon and C. C. Han, Polymer, 2001, 42, 3713-3722.

7 S. Sen, Y. Xie, S. Kumar, H. Yang, A. Bansal, D. Ho, L. Hall, J. Hooper and K. Schweizer, Phys. Rev. Lett., 2007, 98, 128302.

8 A. Tuteja, P. M. Duxbury and M. E. Mackay, Phys. Rev. Lett., 2008, 100, 77801.

9 N. Jouault, F. Dalmas, S. Said, E. Di Cola, R. Schweins, J. Jestin and F. Boué, Macromolecules, 2010, 43, 9881-9891.

10 M. K. Crawford, R. J. Smalley, G. Cohen, B. Hogan, B. Wood, S. K. Kumar, Y. B. Melnichenko, L. He, W. Guise and B. Hammouda, Phys. Rev. Lett., 2013, 110, 196001.

11 K. J. Mutch, J. S. van Duijneveldt and J. Eastoe, Soft Matter, 2007, 3, 155-167.

12 A. Polson, G. Potgieter, J. Largier, G. Mears and F. Joubert, Biochim. Biophys. Acta, 1964, 82, 463-475.

13 T. Odijk, Macromolecules, 1996, 29, 1842-1843.

14 P. G. de Gennes, C. R. Seances Acad. Sci., Vie Acad., 1979, 288, 359-361.

15 R. Sear, Phys. Rev. E: Stat. Phys., Plasmas, Fluids, Relat. Interdiscip. Top., 1998, 58, 724-728.

16 R. Sear, Phys. Rev. Lett., 2001, 86, 4696-4699.

17 P. V. D. Schoot, Macromolecules, 1998, 31, 4635-4638.

18 T. Kramer, S. Scholz, M. Maskos and K. Huber, J. Colloid Interface Sci., 2004, 279, 447-457.

19 T. Kramer, R. Schweins and K. Huber, Macromolecules, 2005, 38, 151-159.

20 T. Kramer, R. Schweins and K. Huber, Macromolecules, 2005, 38, 9783-9793.

21 T. Kramer, R. Schweins and K. Huber, J. Chem. Phys., 2005, 123, 014903.
22 R. S. Ruoff, D. S. Tse, R. Malhotra and D. C. Lorents, J. Phys. Chem., 1993, 97, 3379-3383.

23 H. C. Wong, A. Sanz, J. F. Douglas and J. T. Cabral, J. Mol. Liq., 2010, 153, 79-87.

24 M. E. Mackay, A. Tuteja, P. M. Duxbury, C. J. Hawker, B. Van Horn, Z. Guan, G. Chen and R. S. Krishnan, Science, 2006, 311, 1740-1743.

25 K. A. Barnes, J. F. Douglas, D.-W. Liu and A. Karim, Adv. Colloid Interface Sci., 2001, 94, 83-104.

26 H. C. Wong and J. T. Cabral, Phys. Rev. Lett., 2010, 105, 38301.

27 M. Holmes, M. Mackay and R. Giunta, J. Nanopart. Res., 2007, 9, 753-763.

28 M. A. Yaklin, P. M. Duxbury and M. E. Mackay, Soft Matter, 2008, 4, 2441-2447.

29 H. C. Wong and J. T. Cabral, Macromolecules, 2011, 44, 45304537.

30 H. C. Wong, A. M. Higgins, A. R. Wildes, J. F. Douglas and J. T. Cabral, Adv. Mater., 2012, 25, 985-991.

31 S. Roy, D. Bandyopadhyay, A. Karim and R. Mukherjee, Macromolecules, 2015, 48, 373-382.

32 R. Dattani, K. F. Gibson, S. Few, A. J. Borg, P. A. DiMaggio, J. Nelson, S. G. Kazarian and J. T. Cabral, J. Colloid Interface Sci., 2015, 446, 24-30.

33 K. Huber, S. Bantle, P. Lutz and W. Burchard, Macromolecules, 1985, 18, 1461-1467.

34 M. Daoud, J. P. Cotton, B. Farnoux, G. Jannink, G. Sarma, H. Benoit, C. Duplessix, C. Picot and P. G. de Gennes, Macromolecules, 1975, 8, 804-818.

35 J. P. Cotton, M. Nierlich, F. Boue, M. Daoud, B. Farnoux, G. Jannink, R. Duplessix and C. J. Picot, J. Chem. Phys., 1976, 65, 1101-1108.

36 Q. Ying and B. Chu, Macromolecules, 1987, 20, 362-366.

37 I. Zolotukhin, L. Yanchenko and E. Belonogov, JETP Lett., 1998, 67, 720-722.

38 V. Georgakilas, F. Pellarini, M. Prato, D. M. Guldi, M. MelleFranco and F. Zerbetto, Proc. Natl. Acad. Sci. U. S. A., 2002, 99, 5075-5080.

39 C. Park, H. J. Song and H. C. Choi, Chem. Commun., 2009, 4803-4805.

40 V. J. Anderson and H. N. W. Lekkerkerker, Nature, 2002, 416, 811-815.

41 J. Jeong, W.-S. Kim, S.-I. Park, T.-S. Yoon and B. H. Chung, J. Phys. Chem. C, 2010, 114, 12976-12981. 